\title{
Research Article \\ On the Flow Behavior in Rotor-Stator System with Superposed Flow
}

\author{
Roger Debuchy, Fadi Abdel Nour, and Gérard Bois \\ Laboratoire de Mécanique de Lille, CNRS UMR 8107, Ecole Nationale Supérieure d'Arts et Métiers (ENSAM), \\ 8 Boulevard Louis XIV, 59046 Lille Cedex, France
}

Correspondence should be addressed to Roger Debuchy, roger.debuchy@univ-artois.fr

Received 24 April 2008; Accepted 16 September 2008

Recommended by Akira Murata

\begin{abstract}
The flow between a rotor and a stator at high Reynolds number and small Ekman number is divided into three domains, two boundary layers adjacent to the discs separated by a central core. In the present work, a simple theoretical approach provides analytical solutions for the radial distribution of the core swirl ratio valid for a rotor-stator system with a superposed radial inflow rate. At first, the flow in the rotor boundary layer is assumed to behave as expressed by Owen and Rogers (1989) in the case of a turbulent flow on a rotating single disc. On the stator side, a necessary compensation flow rate must take place according to the conservation of mass. It is found that this compensation flow rate cannot be estimated with a good accuracy using the hypotheses of a stationary disc in a rotating fluid by Owen and Rogers (1989). Thus, two innovative weighting functions are tested, leading to new analytical laws relating the core swirl ratio $K$ to the coefficient of flow rate $C_{q r}$ introduced by Poncet et al. (2005). The adequacy between the theoretical solutions and numerous results of the literature is clearly improved and the discussion allows a better understanding of the flow behavior.
\end{abstract}

Copyright ( 92008 Roger Debuchy et al. This is an open access article distributed under the Creative Commons Attribution License, which permits unrestricted use, distribution, and reproduction in any medium, provided the original work is properly cited.

\section{INTRODUCTION}

Problems involving flows between rotating discs could be representative of a lot of situations often encountered in turbomachinery, such as cooling system of modern gas turbines, pumps, water and steam turbines, and so forth. A detailed review of many recent studies is reported in Owen and Rogers [1].

The earlier studies are proposed by von Karman [2] who simplified the equations of motion to nonlinear differential equations by means of axial symmetry hypothesis for the infinite disc rotating in a quiescent fluid and by Bödewadt [3] who focused on a stationary disc in vicinity of a rotating fluid. Batchelor [4] gathered the two problems in the case of a rotor-stator system and proposed a model based on the presence of two boundary layers adjacent to the discs, and separated by a central core rotating as a solid body. This result has been contrasted by Stewartson [5] who modeled the presence of one boundary layer (the rotor one). Both solutions have been experimented for different cavity aspect ratios and Reynolds numbers.
A lot of numerical and experimental studies dealing with enclosed rotor-stator systems can be found in the literature. Daily and Nece [6] individuated four flow regimes corresponding to laminar or turbulent flow with merged or "separated" boundary layers. Itoh et al. [7] studied the effect of the rotational Reynolds number on the mean velocity field for shrouded discs. It is worth pointing out that, from their studies, the region near the rotating axis remains laminar even for a high rotational rate. Furthermore, the transition from laminar to turbulent regions occurs first along the stator side and then laminar and turbulent flows coexist. Other numerous works dealing with the flow in enclosed systems can be quoted: Stepanoff [8], Szeri et al. [9], Dijkstra and van Heijst [10], Cheah et al. [11], and more recently Randriamampianina et al. [12]. Of course, influence of external flow and peripheral geometry of the cavity is not considered.

In case of a rotor-stator cavity, finite and open at the periphery, peripheral and external conditions are very important. Djaoui et al. [13] and more recently D'Haudt et al. [14] and Debuchy et al. [15] highlighted the strong 
influence of the geometrical dimensionless parameter $\lambda$ defined by the ratio of the difference between stator and rotor radii and the axial gap of the cavity, in the case of an isolated system, that is, without any superposed inflow. The case of a rotor-stator system with a superposed centripetal inflow has been investigated by Debuchy et al. [16]. For high Reynolds number, small gap ratio and small Ekman number, it was found that the flow structure is strongly modified as soon as a very weak inflow is applied, enhancing the level of the core swirl ratio near the axis. Poncet et al. [17, 18] have found an analytical law for the radial distribution of the central core swirl ratio, in very good agreement with numerous experimental and numerical results.

In the present work, a simplified theoretical approach leads to analytical solutions for the core swirl ratio in case of a rotor-stator system with superposed inflow. Starting from results of Owen and Rogers [1], the fluid centrifuged by the rotor wall is assumed to behave as a turbulent flow on a single disc. On the other side (rotor side), it is shown that the compensation flow rate cannot be estimated with a good accuracy using the results of Owen and Rogers [1] in case of a stationary disc in a rotating fluid because a radial circulation of fluid also takes place in the central core. Thus, the authors have tested innovative weighting functions leading to new analytical laws relating the core swirl ratio $K$ to the coefficient of flow rate $C_{q r}$ introduced by Poncet [17]. In the discussion, extensive comparisons with experimental and numerical results are in a very good agreement and provide a better comprehension of the flow behavior.

\section{THEORETICAL APPROACH}

The flow takes place between a rotor of radius $R$, rotating with an angular velocity $\Omega$, and a stationary disc. The axial distance between the two discs is $H$ (Figure 1). The flow is assumed to be incompressible, steady, and axisymmetric. $r$ and $z$ are, respectively, the radial and axial coordinates $(r=0$ at the axis, $z=0$ on the rotor).

The significant dimensionless parameters of the problem are the axial gap ratio of the rotor-stator system $G$, the Reynolds number $R e$, and the combination of both, giving the Ekman number Ek. They are, respectively, defined by

$$
G=\frac{H}{R}, \quad R e=\frac{\Omega R^{2}}{v}, \quad E k=\frac{1}{R e G^{2}} .
$$

Following the theoretical work of Debuchy et al. [16], it is assumed that

$$
G \ll 1, \quad \operatorname{Re} \gg 1, \quad E k \ll 1 .
$$

These later assumptions correspond to a regime with separated boundary layers where convective forces are dominant.

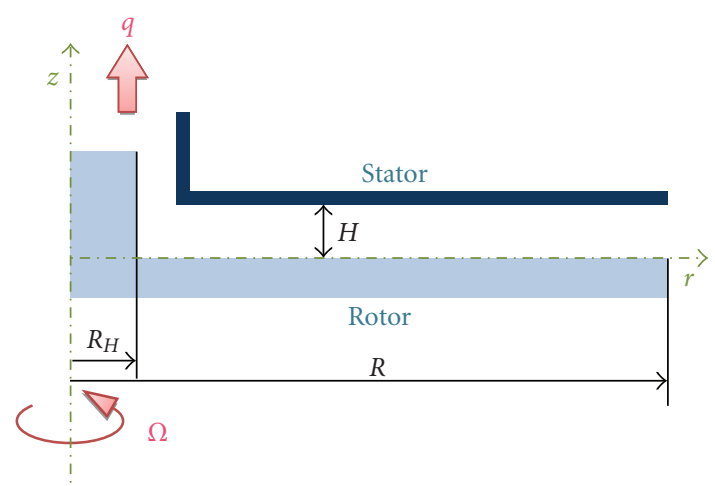

FIGURE 1: Schema of the rotor-stator cavity.

Dimensionless and normalized quantities with superscript $*$ are introduced

$$
\begin{gathered}
r=R r^{*}, \quad z=G R z^{*}, \quad v_{\theta}=\Omega R v_{\theta}^{*}, \quad v_{r}=U_{0} v_{r}^{*}, \\
v_{z}=G U_{0} v_{z}^{*}, \quad p-p_{a t m}=\frac{1}{2} \rho \Omega^{2} R^{2} p^{*} .
\end{gathered}
$$

In rotor-stator cavities with superposed inflow, Debuchy et al. [16] have introduced the following order of magnitude of the radial velocity $U_{0}=\Omega R$.

Substituting (3) in the conservation of mass and momentum equations for laminar flow, the authors obtain

$$
\begin{aligned}
& \frac{1}{r^{*}} \frac{\partial\left(r^{*} v_{r}^{*}\right)}{\partial r^{*}}+\frac{\partial v_{z}^{*}}{\partial z^{*}}=0 \\
& v_{r}^{*} \frac{\partial v_{r}^{*}}{\partial r^{*}}+v_{z}^{*} \frac{\partial v_{r}^{*}}{\partial z^{*}}-\frac{v_{\theta}^{* 2}}{r^{*}}=-\frac{1}{2} \frac{\partial p^{*}}{\partial r^{*}}+\left[E k \frac{\partial^{2} v_{r}^{*}}{\partial z^{* 2}}\right], \\
& v_{r}^{*} \frac{\partial v_{\theta}^{*}}{\partial r^{*}}+v_{z}^{*} \frac{\partial v_{\theta}^{*}}{\partial z^{*}}-\frac{v_{\theta}^{*} v_{r}^{*}}{r^{*}}=\left[E k \frac{\partial^{2} v_{\theta}^{*}}{\partial z^{* 2}}\right], \\
& v_{r}^{*} \frac{\partial v_{z}^{*}}{\partial r^{*}}+v_{z}^{*} \frac{\partial v_{z}^{*}}{\partial z^{*}}=-\frac{1}{2} \frac{1}{G^{2}} \frac{\partial p^{*}}{\partial z^{*}}+\left[E k \frac{\partial^{2} v_{z}^{*}}{\partial z^{* 2}}\right] .
\end{aligned}
$$

In the right-hand side of (5), only the dominant viscous terms have been retained and turbulence has been neglected.

Taking conditions (2) into account, relations (5) become

$$
\begin{gathered}
v_{r}^{*} \frac{\partial v_{r}^{*}}{\partial r^{*}}+v_{z}^{*} \frac{\partial v_{r}^{*}}{\partial z^{*}}-\frac{v_{\theta}^{* 2}}{r^{*}}+\frac{1}{2} \frac{\partial p^{*}}{\partial r^{*}}=0 \\
v_{r}^{*} \frac{\partial v_{\theta}^{*}}{\partial r^{*}}+v_{z}^{*} \frac{\partial v_{\theta}^{*}}{\partial z^{*}}-\frac{v_{\theta}^{*} v_{r}^{*}}{r^{*}}=0 \\
\frac{\partial p^{*}}{\partial z^{*}}=0
\end{gathered}
$$

Relations (4), (6a), (6b), and (6c) have been solved by Debuchy et al. [16]. Two distinct solutions have been obtained: the first one corresponds to a fluid in solid body rotation inside the central core and is valid in the case of an isolated system, that is, without any superposed flow. The second solution is valid in a rotor-stator system with 
superposed inflow $q$. The velocity components are functions of $r^{*}$ and $z^{*}$ :

$$
\begin{aligned}
& v_{r}^{*}=\frac{-c r^{*}}{\sin \alpha} \sin 2 \alpha\left(z^{*}-\frac{1}{2}\right)-\frac{b}{r^{*}} \sin \left(2 \alpha\left(z^{*}-\frac{1}{2}\right)+\beta\right), \\
& v_{\theta}^{*}=\frac{c r^{*}}{\sin \alpha}\left(\cos 2 \alpha\left(z^{*}-\frac{1}{2}\right)-\cos \alpha\right)+\frac{b}{r^{*}} \\
& v_{z}^{*}=\frac{-c}{\alpha \sin \alpha}\left(\cos 2 \alpha\left(z^{*}-\frac{1}{2}\right)-\cos \alpha\right) \\
& p^{*}=b^{2}+c^{2}-c^{2} r^{* 2}-\frac{b^{2}}{r^{* 2}} .
\end{aligned}
$$

As a consequence, the core swirl ratio $K$ defined by $v_{\theta}^{*} / r^{*}$ at mid-gap $\left(z^{*}=1 / 2\right)$ is

$$
K=A_{0} r^{*-2}+B_{0}
$$

$A_{0}$ being function of $q$, and $B_{0}$ being a constant.

In the present work, the authors assume that the order of magnitude of the radial velocity component $U_{0}$ is $q \cdot\left(2 \pi R_{H} H\right)^{-1}, R_{H}$ being the radius of the central hub. This hub which exists in the real machineries allows to avoid the singularity at $r=0$.

This leads to introduce the dimensionless flow rate coefficient defined by

$$
R o_{H}=q \cdot\left(2 \pi \Omega R_{H} R H\right)^{-1}
$$

This later parameter is linked to the Rossby number $R o=$ $q \cdot\left(2 \pi \Omega R^{2} H\right)^{-1}$ introduced by Debuchy et al. [16] by $R o_{H}=$ Ro $\cdot \eta^{-1}$ with $\eta=R_{H} / R$. $\eta$ is assumed to be larger than $10 \%$.

The conservation of mass equation (4) is valid again, whereas relations (5) become

$$
\begin{gathered}
R o_{H}^{2} v_{r}^{*} \frac{\partial v_{r}^{*}}{\partial r^{*}}+R o_{H}^{2} v_{z}^{*} \frac{\partial v_{r}^{*}}{\partial z^{*}}-\frac{v_{\theta}^{* 2}}{r^{*}}=-\frac{1}{2} \frac{\partial p^{*}}{\partial r^{*}}+\left[R o_{H} E k \frac{\partial^{2} v_{r}^{*}}{\partial z^{* 2}}\right], \\
R o_{H}^{2} v_{r}^{*} \frac{\partial v_{\theta}^{*}}{\partial r^{*}}+R o_{H}^{2} v_{z}^{*} \frac{\partial v_{\theta}^{*}}{\partial z^{*}}-R o_{H}^{2} \frac{v_{\theta}^{*} v_{r}^{*}}{r^{*}}=\left[R o_{H} E k \frac{\partial^{2} v_{\theta}^{*}}{\partial z^{* 2}}\right], \\
R o_{H}^{2} v_{r}^{*} \frac{\partial v_{z}^{*}}{\partial r^{*}}+R o_{H}^{2} v_{z}^{*} \frac{\partial v_{z}^{*}}{\partial z^{*}}=-\frac{1}{2} \frac{1}{G^{2}} \frac{\partial p^{*}}{\partial z^{*}}+\left[R o_{H} E k \frac{\partial^{2} v_{z}^{*}}{\partial z^{* 2}}\right] .
\end{gathered}
$$

Taking conditions (2) into account, $R o_{H} \sim 1$ leads to relations (5), otherwise when $R o_{H} \ll 1$, relation (6c) is valid again while the radial equilibrium (6a) is to be replaced by

$$
\frac{v_{\theta}^{* 2}}{r^{*}}=\frac{1}{2} \frac{d p^{*}}{d r^{*}} .
$$

The relevant conclusion is that the pressure $p^{*}$ and tangential velocity $v_{\theta}^{*}$ are independent of $z^{*}$, but no analytical solution could be obtained from relation (11) only. The radial and axial velocity components are linked together by the conservation of mass equation (4). The following theoretical development is based on continuity considerations in order to provide additional information about the radial evolution of $p^{*}$ and $v_{\theta}^{*}$.

On the rotor side, a volume flow rate $q_{R}$ induced by centrifugal effect takes place inside the boundary layer. When a superposed inflow rate $q$ is extracted, the equation of conservation of mass, using the dimensionless volume flow rates denoted by superscripts $*$ and defined by $q^{*}=q / v r$, $q_{C}^{*}=q_{C} / v r$, and $q_{R}^{*}=q_{R} / v r$, is

$$
q_{R}^{*}-q^{*}+q_{C}^{*}=0
$$

where $q_{C}^{*}$ is called the dimensionless compensation flow rate. Noting that the sign of the term $q_{C}^{*}$ could not be determined a priori.

On the rotor side, the authors assume that $q_{R}^{*}$ corresponds to the dimensionless flow rate entrained on a single rotating disc when the flow is turbulent. Following the results of Owen and Rogers, $q_{R}^{*}$ is proportional to $R e_{r}^{4 / 5}, R e_{r}$ being the local Reynolds number. On the other side, when considering the stator as a stationary disc in a rotating fluid, the dimensionless flow rate in the case of turbulent flow is proportional to $R e_{\omega}^{4 / 5}$. Here, $R e_{\omega}$ is the local Reynolds number based on the velocity of the rotating fluid in the central core. Introducing the core swirl ratio defined by $K=v_{\theta}^{*} / r^{*}$ outside the boundary layers, the authors obtain $R e_{\omega}=K R e_{r}$ and consequently

$$
q_{R}^{*} \propto\left(r^{* 2} R e\right)^{4 / 5}, \quad q_{C}^{*} \propto K^{4 / 5}\left(r^{* 2} R e\right)^{4 / 5} .
$$

The combination of relations (12) and (13) leads to

$$
K=\left[\begin{array}{ll}
A_{1} & r^{*-13 / 5}+B_{1}
\end{array}\right]^{4 / 5}
$$

with $A_{1}$ being proportional to $q$, and $B_{1}$ being a constant.

This relation is valid under the assumption that the compensation flow rate behaves as in the boundary layer of the stationary disc in a rotating fluid. The first term in the right side of relation (14) is linked to the presence of a superposed flow because $A_{1}$ is proportional to the flow rate $q$. Following Poncet et al. [17] and introducing the local coefficient of flow rate $C_{q r}=q R e_{r}^{1 / 5} /\left(2 \pi \Omega r^{3}\right)$, relation (14) becomes

$$
K=\left[\begin{array}{ll}
a_{1} & C_{q r}+B_{1}
\end{array}\right]^{5 / 4}
$$

with $a_{1}$ and $B_{1}$ being constants.

As soon as there is a radial circulation of fluid outside boundary layers, which may occur when there is a superposed radial inflow, the second relation of (13) is no longer valid. Consequently, the authors propose a correction by using a weighting function, so that (13) is to be replaced by

$$
q_{R}^{*} \propto\left(r^{* 2} R e\right)^{4 / 5}, \quad q_{C}^{*} \propto W K^{4 / 5}\left(r^{* 2} R e\right)^{4 / 5} .
$$

Since the flow behavior in a rotor-stator system is closely linked to the level of the core swirl ratio and to the local coefficient of flow rate, the authors foresee to test two types of weighting function.

The first one is $W=K^{c}$. Starting from Owen and Rogers results [1] in case of laminar flow, hypotheses (13) have to be 
written with a power of $1 / 2$ instead of $4 / 5$. The combination of relations (12) and (13) leads to the following analytical relation: $K=\left[\begin{array}{ll}A_{1} & r^{*-2}+B_{1}\end{array}\right]^{2}$. This solution, which is widely different from (8), cannot be in good agreement with the experimental results of Debuchy et al. [16]. When introducing a weighting function of the form $W=K^{c}$, the compensation dimensionless volume flow rate becomes $q_{C}^{*} \propto K^{c+1 / 2}\left(r^{* 2} R e\right)^{1 / 2}$ and solution (8) can be obtained with $c=1 / 2$. According to previous remark, the authors foresee that introducing a weighting function of the form $W=K^{c}$ in the second relation of (16) should also improve assumption (13) in case of turbulent flow. Consequently, (14) and (15) are to be replaced by

$$
K=\left[\begin{array}{ll}
A_{1} & r^{*-13 / 5}+B_{1}
\end{array}\right]^{1 / c}, \quad K=\left[\begin{array}{ll}
a_{1} & C_{q r}+B_{1}
\end{array}\right]^{1 / c}
$$

with $A_{1}$ being proportional to $q, a_{1}$ and $B_{1}$ being constants.

The second weighting function is chosen with the form of $W=f\left(C_{q r}\right)$ because of the physical properties of rotorstator flow: circulation of fluid outside boundary layers must occur when superposed flow rate $q$ increases, and/or radial position $r$ decreases. The weighting function must also verify $f\left(C_{q r}\right) \rightarrow 1$ when $C_{q r} \rightarrow 0$ in order to be adequate with the original hypothesis (13). Relations (14) and (15) are to be replaced by

$$
K=\left[\frac{A_{1} r^{*-13 / 5}+B_{1}}{f\left(A_{1} r^{*-13 / 5}\right)}\right]^{5 / 4}, \quad K=\left[\frac{a_{1} C_{q r}+B_{1}}{f\left(C_{q r}\right)}\right]^{5 / 4}
$$

with $A_{1}$ being proportional to $q, a_{1}$ and $B_{1}$ being constants.

The adequacy between relations (14), (15), (17), and (18), and numerous theoretical, experimental, and numerical results of the literature will be discussed in the following section.

\section{RESULTS AND DISCUSSION}

The validity of the theory is now discussed by making comparisons with the experimental results of Debuchy [19], Debuchy et al. [16], and also with the theoretical, numerical, and experimental works of Poncet [17], Poncet et al. [18, 20]. All results have been obtained in an annular cavity between a rotating disk equipped with a central hub and a stationary disk with a central outlet section in order to ensure a superposed flow. The two experimental setups have already been detailed in Debuchy [19] and Poncet [17]. The most significant differences are located at the periphery of the two systems, with the consequence that it may influence the inlet flow properties. It has been shown by Debuchy et al. [15] that if the inlet flow comes from the external fluid at rest, then the peripheral core swirl ratio (i.e., the preswirl level ) is weak, otherwise the preswirl will be higher. Consequently the authors are more interested in the description of the peripheral geometry of the two cavities (Figures 2(a) and 2(b)).

The apparatus used by Debuchy [19] was totally open at the periphery. The rotor, $750 \mathrm{~mm}$ in diameter, was rotating

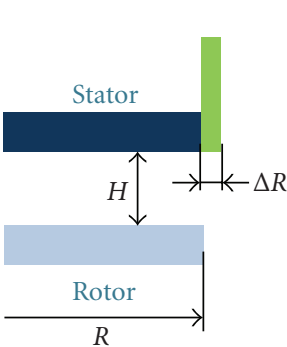

(a) Debuchy [19]

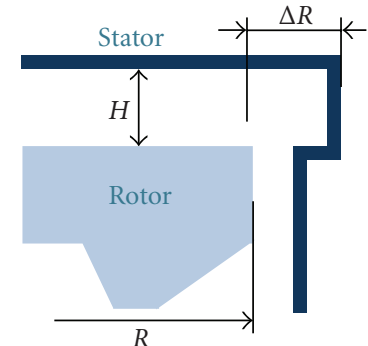

(b) Poncet [17]
FIgURE 2: Schema of the peripheral inlet conditions.

\begin{tabular}{|c|c|c|c|}
\hline & \multirow{2}{*}{$\begin{array}{c}\text { Debuchy [19] } \\
\text { Test case A }\end{array}$} & \multicolumn{2}{|c|}{ Poncet [17] } \\
\hline & & Test case B & Test case $\mathrm{C}$ \\
\hline$R$ & $375 \mathrm{~mm}$ & \multicolumn{2}{|c|}{$250 \mathrm{~mm}$} \\
\hline$H$ & $30 \mathrm{~mm}$ & \multicolumn{2}{|c|}{$9 \mathrm{~mm}$} \\
\hline$\Delta R$ & $8 \mathrm{~mm}$ & \multicolumn{2}{|c|}{$3 \mathrm{~mm}$} \\
\hline$\eta$ & 0.24 & \multicolumn{2}{|c|}{0.15} \\
\hline$\lambda$ & 0.27 & \multicolumn{2}{|c|}{0.33} \\
\hline$G$ & 0.08 & \multicolumn{2}{|c|}{0.036} \\
\hline $\operatorname{Re}$ & $1.47 \times 10^{6}$ & \multicolumn{2}{|c|}{$10^{6}$} \\
\hline$E k$ & $1.06 \times 10^{-4}$ & \multicolumn{2}{|c|}{$7.7 \times 10^{-4}$} \\
\hline$R o_{H}$ & $6.95 \times 10^{-2}$ & $17.4 \times 10^{-2}$ & $29.2 \times 10^{-2}$ \\
\hline$C_{w}$ & 11886 & 5929 & 9881 \\
\hline & Air & \multicolumn{2}{|c|}{ Water } \\
\hline & Hot wire anemometry & \multicolumn{2}{|c|}{$\begin{array}{l}\text { Laser doppler anemometry } \\
\text { LDA }\end{array}$} \\
\hline
\end{tabular}

TABLE 1

at $1500 \mathrm{rev} / \mathrm{min}$ and equipped with a central hub, $180 \mathrm{~mm}$ in diameter. The axial gap of the cavity was fixed to $30 \mathrm{~mm}$ which means that the gap ratio was 0.08 . A stationary shroud, $8 \mathrm{~mm}$ in thickness, covered the axial upper side of the stator, which made the stator radius larger than that of the rotor. Consequently, the $\lambda$ parameter introduced by Debuchy et al. [15] was fixed to 0.27 . Measurements were performed in air at Reynolds number fixed to $1.47 \times 10^{6}$ (i.e., Ekman number fixed to $1.06 \times 10^{-4}$ ).

In the rotor-stator system used by Poncet, an external stationary shroud near the periphery forced the fluid to recirculate near the rotor with the consequence that the preswirl level is high. The rotor, $500 \mathrm{~mm}$ in diameter, was rotating up to $600 \mathrm{rev} / \mathrm{min}$. The axial gap of the cavity was adjustable between 1 and $12 \mathrm{~mm}$. Measurements were performed in water using a laser doppler anemometry.

The main characteristics and dimensionless parameter values retained for the discussion section are listed in Table 1. In all cases, the conditions (2) are always verified. The values of $\lambda$ parameter are to a large extent identical in the two experimental setups. In test case $\mathrm{A}, R o_{H}$ is much lower than 1 , on the contrary to test cases $\mathrm{B}$ and $\mathrm{C}$. However, $R o_{H}^{2}$ is always lower than $1\left(R o_{H}^{2} \approx 8.5 \times 10^{-2}\right.$ is the maximum value for the test case $C)$, which means that relation (11) is valid and that the solutions (14), (15), (17), and (18) are to be tested. 


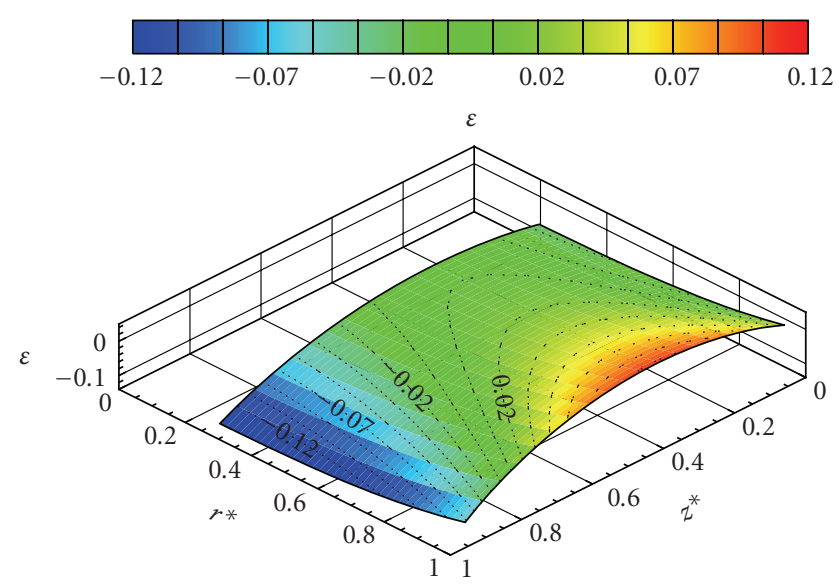

FIgure 3: Axial and radial distribution of $\varepsilon$ in test case A.

In the first part of the discussion, the authors evaluate the order of magnitude of the terms neglected in relation (11) by comparison to (6a), that is, the order of magnitude of $v_{r}^{*}\left(\partial v_{r}^{*} / \partial r^{*}\right)+v_{z}^{*}\left(\partial v_{r}^{*} / \partial z^{*}\right)$ with regard to $v_{\theta}^{* 2} / r^{*}$. The quantity $\varepsilon$ is used to define the ratio $\left(v_{r}^{*}\left(\partial v_{r}^{*} / \partial r^{*}\right)+\right.$ $\left.v_{z}^{*}\left(\partial v_{r}^{*} / \partial z^{*}\right)\right) /\left(v_{\theta}^{* 2} / r^{*}\right)$.

The experimental results of test case A have already been compared to solution (7) with values of $b, c, \alpha$ and $\beta$ adjusted with the experimental results of Debuchy et al. [16]. The ratio $\varepsilon$ is computed from relation (7) with $b=0.175, c=$ $0.04, \alpha=0.3$, and $\beta=0.09$. The obtained results are plotted in Figure 3 versus $r^{*}$ and $z^{*}$ for $0.24 \leq r^{*} \leq 1.0$ and $0.05 \leq z^{*} \leq 0.95$. Boundary layers have not been considered because the theoretical solution is valid only in the central core: viscous terms in the right-hand of relation (6a), (6b), and $(6 \mathrm{c})$ have been eliminated, so that the no-slip conditions $v_{r}^{*}=0$ at $z^{*}=0$ and $z^{*}=1, v_{\theta}^{*}=r^{*}$ at $z^{*}=0$, and $v_{\theta}^{*}=0$ at $z^{*}=1$ were not satisfied in the theoretical solution. As shown in Figure 3, the absolute value of the ratio $\varepsilon$ is always below $13 \%$, the maximum being located on the stator side for the smallest value of $r^{*}$.

For test cases B and C, which have not yet been compared to solution (7), constants $b$ and $c$ have been adjusted firstly starting from the dimensionless pressure radial distribution (Figure 4). The authors mention that the dimensionless pressure $p^{*}$ is a little bit shifted in order to satisfy the condition $p^{*}=0$ at $r^{*}=1$ instead of the condition $\left\{p^{*}-p^{*}\left(r^{*}=0.92\right)=0\right\}$ chosen by Poncet et al. [18]. In a second step, the constants $\alpha$ and $\beta$ are adjusted from the radial and tangential velocity profiles (Figure 5). The obtained values are mentioned in Table 2. For test cases B and $\mathrm{C}$, the constants adjustment is very difficult because the authors only have few experimental results. However, the tangential velocity levels (Figures $5(\mathrm{a})$ and $5(\mathrm{~b})$ ) and the centripetal flow tendency for the radial velocity profiles at $r^{*}=0.56$ (Figures 5(c) and 5(d)) are in good agreement with the theoretical solution.

Using the adjusted constants in Table 2, the authors have found a good agreement for the radial distributions of the core swirl ratio, except the peripheral zone where the theoretical preswirl level is slightly underestimated. As for

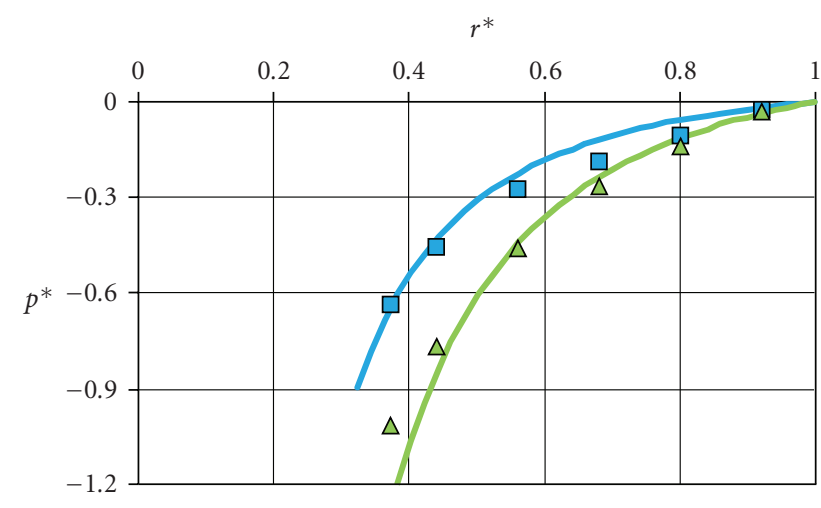

Experimental data test case B

$\Delta$ Experimental data test case $\mathrm{C}$

Model in (7) test case B

Model in (7) test case C

FIgURE 4: Radial pressure distribution.

TABLE 2

\begin{tabular}{lcccc}
\hline & $b$ & $c$ & $\alpha$ & $\beta$ \\
\hline Test case B & 0.32 & 0.04 & 0.25 & 0.82 \\
Test case C & 0.45 & 0.04 & 0.25 & 0.93 \\
\hline
\end{tabular}

test case $\mathrm{A}$, the radial and axial evolution of the $\varepsilon$ ratio is plotted for $0.15 \leq r^{*} \leq 1.0$ and $0.05 \leq z^{*} \leq 0.95$ (Figures 7 and 8 ). It is found again that this ratio is below $13 \%$.

The conclusion of the first part of the discussion is that the simplified radial momentum equation (11) can be used instead of relation (6a) since the ratio $\varepsilon$ is small. The Rossby number defined in relation (9) is a significant parameter because $\varepsilon$ is small for $R o_{H}^{2} \ll 1$. The analytical solutions (14), (15), (17), and (18) in the theoretical part, based on (9) and (11) can be tested in the following part of this discussion.

To begin, the validity of the hypothesis (13) which leads to the solutions (14) or (15) is tested. For this purpose, comparisons between the theoretical solution (15) and results extracted from Poncet et al. [18] are presented in Figure 9. Poncet et al. [18] have found a very good agreement between their analytical law $K=2\left[a C_{q r}+b\right]^{5 / 7}-1$ and numerous experimental results (with the adjusted values $a \approx$ 5.9 and $b \approx 0.63$ ) and also with numerical results based on RSM model (with an adjusted value of $a$ slightly lower: $a \approx 5.3$ ).

Testing relation (15), the best results were obtained for $a_{1} \approx 6.7$ and $B_{1} \approx 0.52$ in the case of experiments and $a_{1} \approx$ 6.2 and $B_{1} \approx 0.52$ for numerical results. However, the authors found that the agreement is to be improved. The last remark had led the authors to insert a weighting function in (16) and to test relations (17) and (18).

Considering the weighting function $W=K^{c}$, the best agreement between relation (17) and the experimental, numerical, and theoretical results of the literature has been 


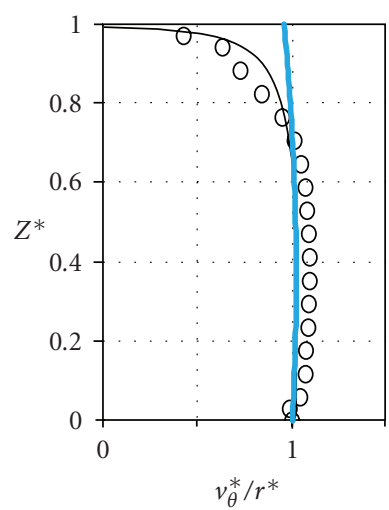

O Measurement data

— IRPHE's numerical results

_ Model in (7) test case B

(a) Test case B

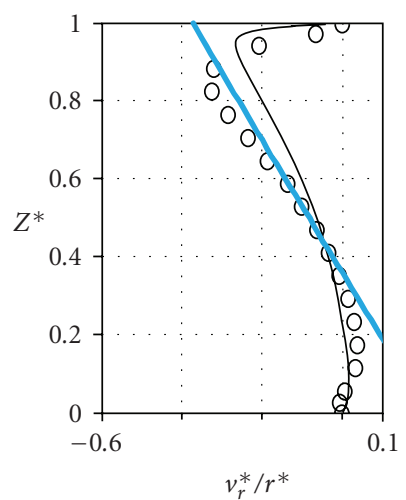

O Measurement data

— IRPHE's numerical results

_ Model in (7) test case B

(c) Test case B

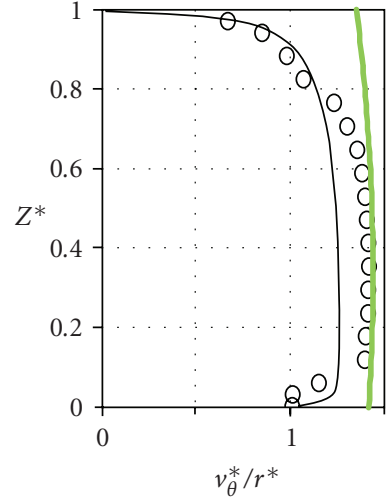

O Measurement data

— IRPHE's numerical results - Model in (7) test case C

(b) Test case $\mathrm{C}$

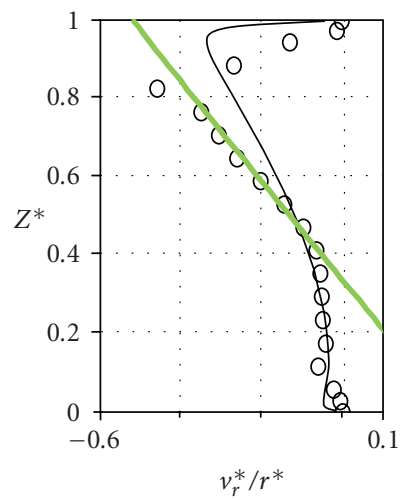

O Measurement data

— IRPHE's numerical results _ Model in (7) test case C

(d) Test case C

Figure 5: Tangential ((a) and (b)) and radial ((c) and (d)) velocity profiles.

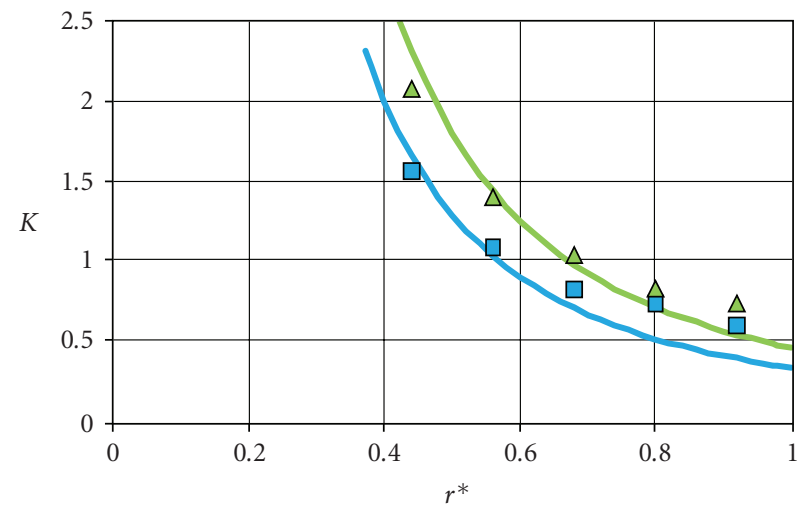

․ Experimental data test case B

$\triangle$ Experimental data test case $\mathrm{C}$

— Model in (7) test case B

_ Model in (7) test case C

FIGURE 6: Core swirl ratio distribution.

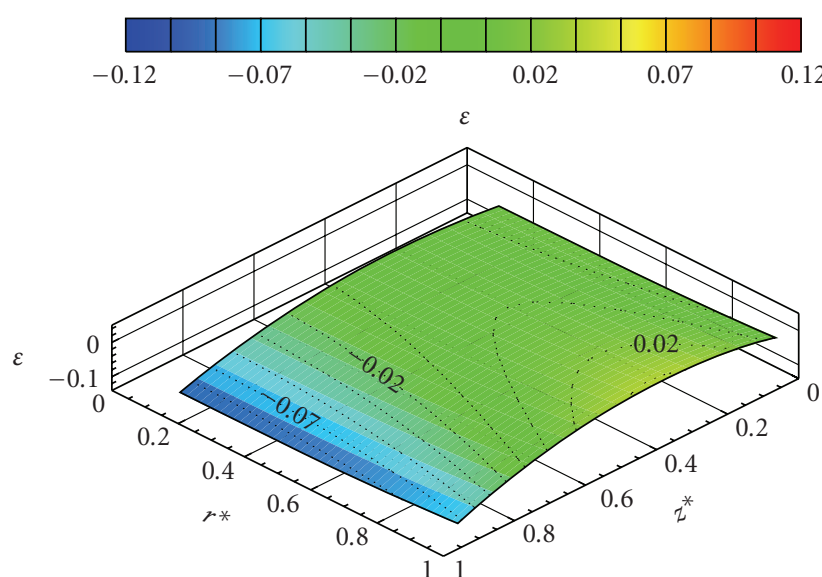

FIgURE 7: $\varepsilon$ axial and radial distribution in the test case B.
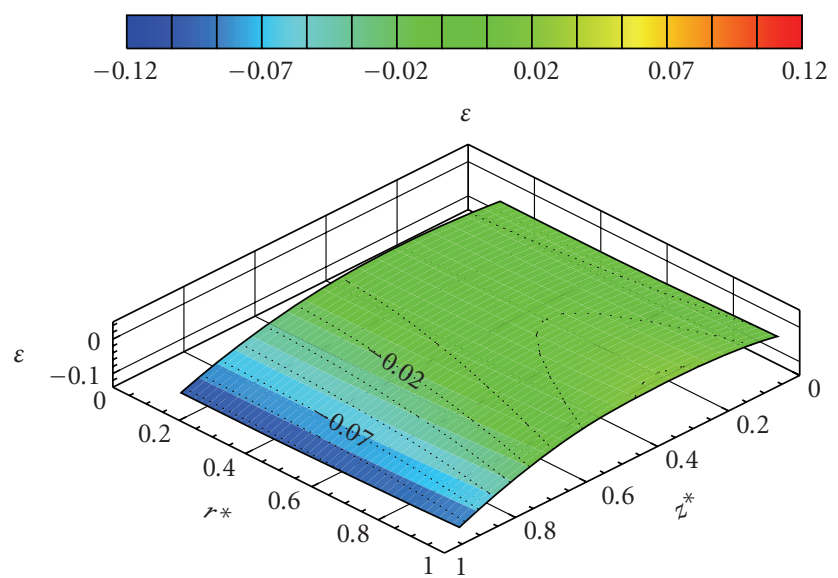

FIGURE 8: $\varepsilon$ axial and radial distribution in the test case C.

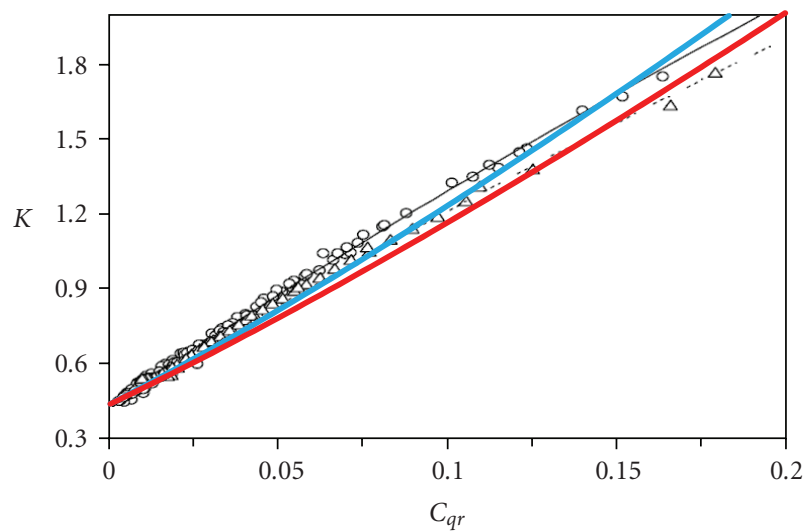

- Experimental data

$\triangle$ Numerical data

— IRPHEs model with experimental data

.... IRPHEs model with numerical data

- Relation (15) with $a_{1} \approx 6.7$ and $B_{1} \approx 0.52$

Relation (15) with $a_{1} \approx 6.2$ and $B_{1} \approx 0.52$

FIgURE 9: Core swirl ratio distribution deduced from (15). Comparison with experimental and numerical results from Poncet [17]. 


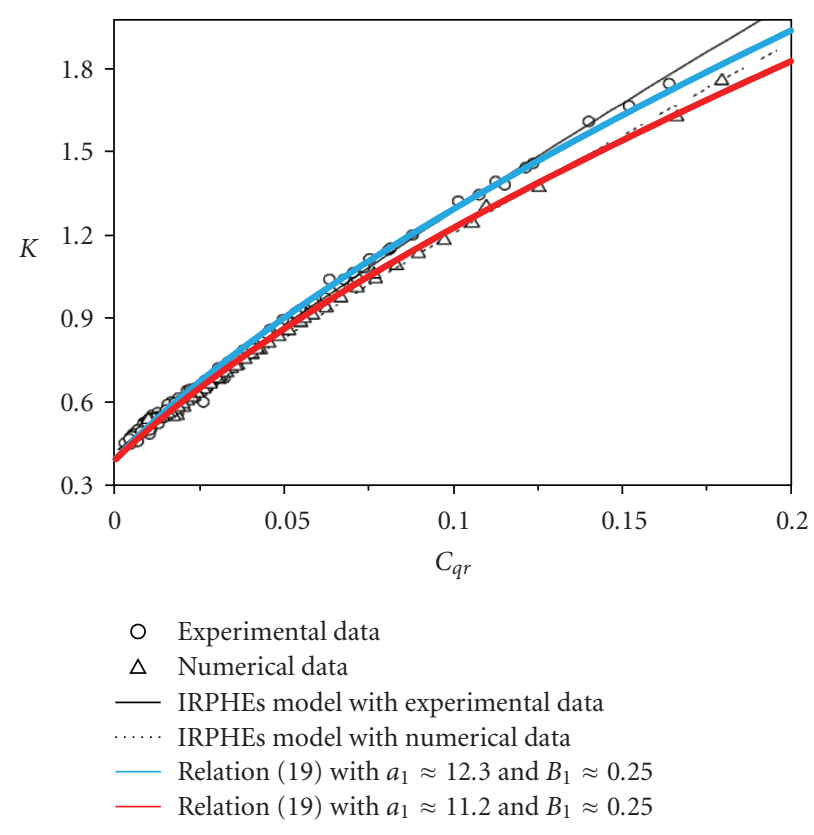

FIgUre 10: Core swirl ratio distribution deduced from (19). Comparison with experimental and numerical results from Poncet [17].

found for $c=3 / 2$. Consequently, relation (17) is to be replaced by

$$
K=\left[\begin{array}{ll}
A_{1} & r^{*-13 / 5}+B_{1}
\end{array}\right]^{2 / 3}, \quad K=\left[\begin{array}{ll}
a_{1} & C_{q r}+B_{1}
\end{array}\right]^{2 / 3} .
$$

The second relation in (19) is validated using the experimental and numerical results from Poncet et al. [18] after adjustment of constants $a_{1}$ and $B_{1}\left(a_{1} \approx 12.3\right.$ and $B_{1} \approx 0.25$ for comparison with experiments; $a_{1} \approx 11.2$ and $B_{1} \approx 0.25$ for comparison with numerical results). The agreement is much better than that obtained with relation (15), at least for $C_{q r} \leq 0.2$ (Figure 10).

The first relation in (19) giving the radial distribution of the core swirl ratio is validated using the experimental results of test cases $\mathrm{A}, \mathrm{B}$, and $\mathrm{C}$. A very good agreement has been found with $A_{1} \approx 0.1$ and $B_{1} \approx 0.03$ for test case $\mathrm{A}, A_{1} \approx 0.18$ and $B_{1} \approx 0.25$ for test case $\mathrm{B}$, and $A_{1} \approx 0.30$ and $B_{1} \approx 0.25$ for test case $C$ for both the core swirl ratio (Figure 11) and the dimensionless pressure (Figure 12) distributions.

As $c=3 / 2$, the weighting function introduced in (16) is $K^{7 / 10}$, which is found to be close to the weighting function $K^{1 / 2}$ mentioned in the theoretical approach in case of laminar boundary layer.

Large discrepancies are observed in the core swirl ratio distribution for $C_{q r} \geq 0.35$ whereas the analytical solution from Poncet [17] is valid for values of $C_{q r}$ below 0.2 as shown in Figure 13. This may be improved with a weighting function of $C_{q r}$ in (16), as previously discussed, or with a more realistic hypothesis concerning the rotor boundary layer. Indeed, it is well known that the rotor flow rate is centripetal for core swirl ratio upper than 1: this behavior is not taken into account in hypothesis (13).

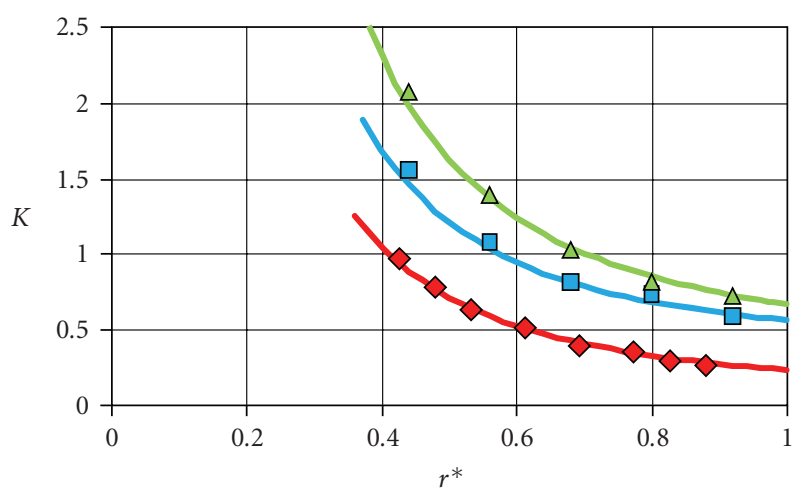

$\diamond$ Experimental data for test case A

$\square \quad$ Experimental data for test case B

$\triangle$ Experimental data for test case $\mathrm{C}$

Test case A: $A_{1} \approx 0.1$ and $B_{1} \approx 0.03$

Test case $\mathrm{B}: A_{1} \approx 0.18$ and $B_{1} \approx 0.25$

Test case C: $A_{1} \approx 0.3$ and $B_{1} \approx 0.25$

FIgure 11: Core swirl ratio distribution.

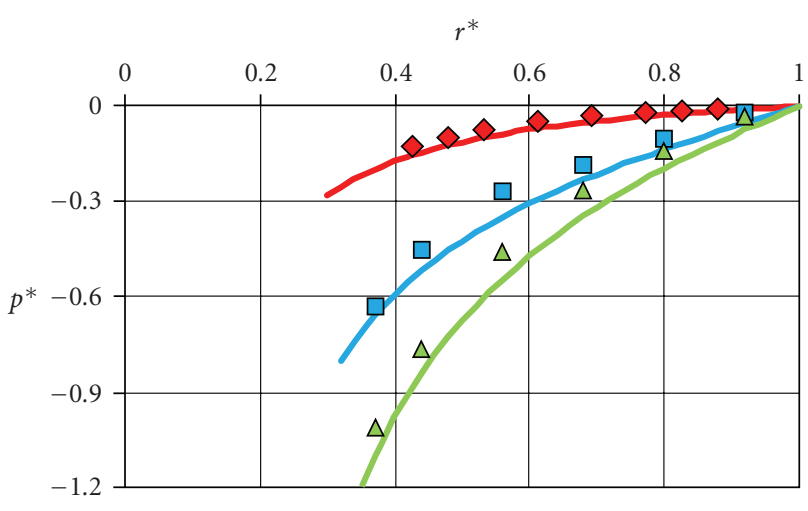
$\diamond$ Experimental data for test case A
$\square$ Experimental data for test case $\mathrm{B}$
$\triangle$ Experimental data for test case $\mathrm{C}$
Test case A: $A_{1} \approx 0.1$ and $B_{1} \approx 0.03$
- Test case B: $A_{1} \approx 0.18$ and $B_{1} \approx 0.25$
Test case C: $A_{1} \approx 0.3$ and $B_{1} \approx 0.25$

FIGURE 12: Radial pressure distribution computed with (11).

When testing the weighting function $W=f\left(C_{q r}\right)$, the best agreement between relation (18) and the theoretical, experimental, and numerical results of the literature has been obtained for $W=e^{\phi C_{q r}}$. As previously discussed, this weighting function has been chosen because $W \rightarrow 1$ when $\mathrm{C}_{q r} \rightarrow 0$, which means that assumption (13) is also valid in the case of rotor-stator system without any superposed flow. Consequently the relation (18) becomes

$$
K=\left[\frac{A_{1} r^{*-13 / 5}+B_{1}}{e^{\phi r^{*-13 / 5}}}\right]^{5 / 4}, \quad K=\left[\frac{a_{1} C_{q r}+B_{1}}{e^{\varphi C_{q r}}}\right]^{5 / 4}
$$

$\phi$ being proportional to $q$ and $\varphi$ being constants. 


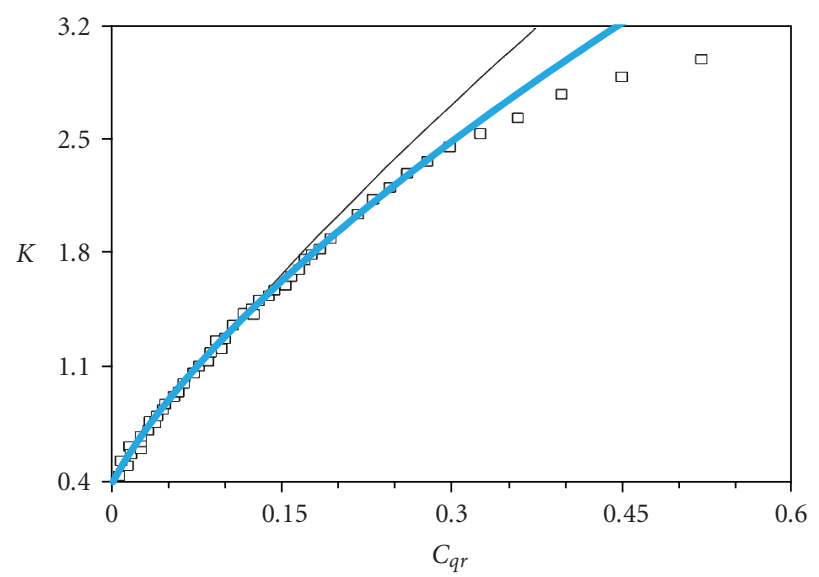

$\square \quad$ IRPHE experimental data without post swirl
$-\quad$ IRPHE model
- Relation (19) with $a_{1} \approx 12.3$ and $B_{1} \approx 0.25$

FIgure 13: Core swirl ratio distribution deduced from (19). Comparison with experimental and numerical results from Poncet [17].

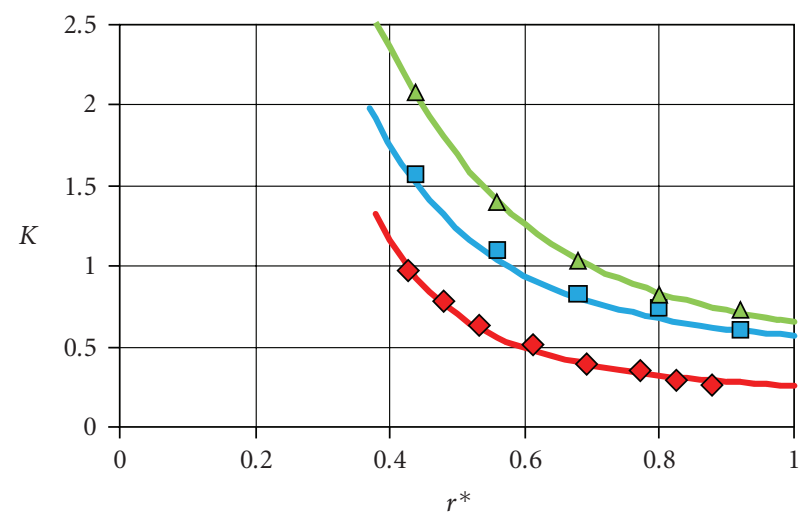

$\diamond$ Experimental data for test case $\mathrm{A}$
$\square \quad$ Experimental data for test case $\mathrm{B}$
$\triangle \quad$ Experimental data for test case $\mathrm{C}$
Test case $\mathrm{A}: A_{1} \approx 0.085, B_{1} \approx 0.254$ and $\varphi \approx 0.004$
Test case $\mathrm{B}: A_{1} \approx 0.143, B_{1} \approx 0.5$ and $\varphi \approx 0.024$
Test case $\mathrm{C}: A_{1} \approx 0.238, B_{1} \approx 0.5$ and $\varphi \approx 0.04$

FIgURE 14: Core swirl ratio distribution.

Comparisons between the theoretical law (20) and the experimental data corresponding to test case $\mathrm{A}$ (with $A_{1} \approx$ $0.085, B_{1} \approx 0.254$, and $\phi \approx 0.004$ ), test case $\mathrm{B}$ (with $A_{1} \approx$ $0.143, B_{1} \approx 0.500$, and $\phi \approx 0.024$ ), and test case $C$ (with $A_{1} \approx 0.238, B_{1} \approx 0.500$, and $\phi \approx 0.04$ ) are shown in Figures 14 and 15 . The results are in very good agreement.

Finally, the validity of the second equation of (20) has been tested over the complete positive range of $C_{q r}$. Taking Figure 13 as a reference, one additional configuration has been considered: it consists of the rotor-stator system in Figure 2(b) with a central hub generating another value of post-swirl (i.e., the central core swirl ratio near the hub). Of
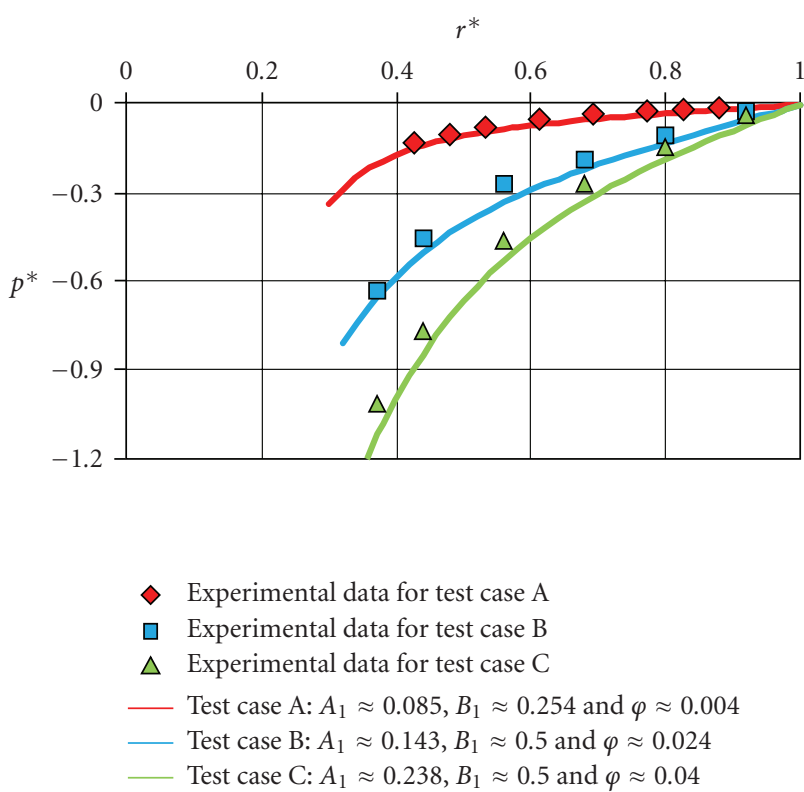

FIGURE 15: Radial pressure distribution computed with (11).

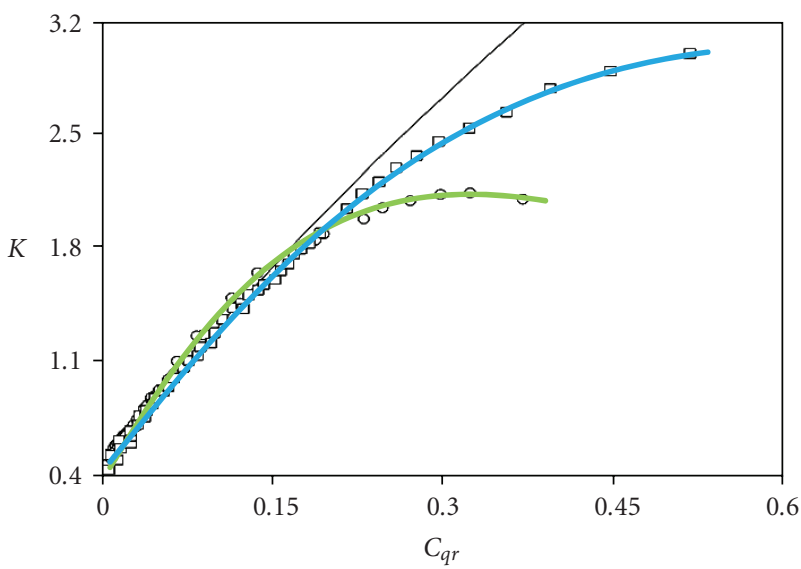

$\square \quad$ IRPHE experimental data without post swirl

O IRPHE experimental data with post swirl

— IRPHE model

- Relation (20): $a_{1} \approx 8.85$ and $B_{1} \approx 0.5$ and $\varphi \approx 1.45$

- Relation (20): $a_{1} \approx 12.4$ and $B_{1} \approx 0.46$ and $\varphi \approx 2.77$

FIgure 16: Core swirl ratio distribution deduced from (20). Comparison with experimental and numerical results from Poncet [17].

sure, the constants in the analytical law need to be adjusted for each configuration. An excellent agreement between theory and experiments is shown in Figure 16 for $C_{q r}$ values up to "0.6."

\section{CONCLUSION}

The aim of this work was to propose a simple theoretical approach in order to obtain a new law relating the core swirl ratio $K$ to the dimensionless flow coefficient $C_{q r}$ in 
a rotor-stator system with superposed flow. Starting from the published results of Owen and Rogers, the authors assumed that the fluid on the rotor side behaves as a turbulent flow on a rotating single disc. It is shown that the compensation flow rate on the stator side cannot be described when using the hypothesis of a stationary wall in a rotating fluid by Owen and Rogers but that an innovative weighting function is to be introduced. At first, testing a weighting function with the form of $W=K^{c}$ is interesting because it leads to well-known solutions of the literature. The adequacy between theory and numerous published data is good for a limited range of the coefficient of flow rate. This result is clearly improved with a weighting function with the form of $W=e^{\phi C_{q r}}$. It leads to a new explicit analytical solution for $K$ valid over a wide range of the $C_{q r}$ coefficient, knowing that the radial distribution of the core swirl ratio is an interesting quantity for turbomachinery applications.

\section{NOMENCLATURE}

$C_{w}:$ Dimensionless flow rate $=q / v R$

$C_{q r}$ : Coefficient of flow rate $=q R e_{r}^{1 / 5} /\left(2 \pi \Omega r^{3}\right)$

Ek: $\quad$ Ekman number $=\left(\operatorname{Re} G^{2}\right)^{-1}$

$G$ : $\quad$ Gap ratio $=H / R$

$H$ : Axial gap of the cavity

$K: \quad$ Core swirl ratio $=v_{\theta}^{*} / r^{*}$ at $z^{*}=1 / 2$

$p: \quad$ Static pressure on the stator

$p^{*}$ : Dimensionless static pressure on the stator $=\left(p-p_{a t m}\right) /\left((1 / 2) \rho \Omega^{2} R^{2}\right)$

$p_{\text {atm }}$ : Atmospheric pressure

$q$ : Superposed volume flow rate

$q^{*}$ : Local dimensionless flow rate $=q / v r$

$r$ : Radial coordinate

$r^{*}: \quad$ Dimensionless radial distance $=r / R$

$R: \quad$ Outer radius of the rotor

$R_{H}$ : Outer radius of the hub

Re: $\quad$ Reynolds number $=\Omega R^{2} / v$

$R e_{r}$ : Local Reynolds number $=\Omega r^{2} / v$

$R o_{H}:$ Rossby number $=q /\left(2 \pi \Omega R_{H} R H\right)$

$U_{0}$ : Order of the magnitude of radial velocity

$v_{r}$ : Radial velocity

$v_{\theta}$ : Tangential velocity

$v_{z}:$ Axial velocity

$v_{r}^{*}:$ Dimensionless radial velocity $=v_{r} / U_{0}$

$v_{\theta}^{*}:$ Dimensionless tangential velocity $=v_{\theta} / \Omega R$

$v_{z}^{*}:$ Dimensionless axial velocity $=v_{z} /\left(G U_{0}\right)$

$z$ : Axial distance from the wall of the rotor inside the cavity

$z^{*}$ : Dimensionless axial distance from the wall of the rotor inside the cavity $=z / H$

$\Delta R: \quad$ Difference between stator and rotor radii

$\lambda: \quad$ Dimensionless geometrical parameter $=\Delta R / H$

$\eta: \quad$ Dimensionless geometrical parameter $=R_{H} / R$

$\Omega: \quad$ Angular speed of the rotor

$\rho:$ Density

$v$ : Cinematic viscosity.

\section{SUBSCRIPTS}

$C$ : Used for the compensation flow rate

$R$ : Used for the flow rate of the rotor boundary layer.

\section{REFERENCES}

[1] J. M. Owen and R. H. Rogers, "Flow and heat transfer in rotating-disc system," in Rotor-Stator System, W. D. Morris, Ed., vol. 1, pp. 93-124, John Wiley \& Sons, New York, NY, USA, 1989.

[2] T. von Kármán, "Uber laminare und turbulente reibung," Zeitschrift für Angewandte Mathematik und Mechanik, vol. 1, no. 4, pp. 233-252, 1921.

[3] U. T. Bödewadt, "Die Dehströmung uber festem Grunde," Zeitschrift für Angewandte Mathematik und Mechanik, vol. 20, pp. 241-253, 1940.

[4] G. K. Batchelor, "Note on a class of solutions of the NavierStokes equations representing steady rotationally-symmetric flow," The Quarterly Journal of Mechanics and Applied Mathematics, vol. 4, no. 1, pp. 29-41, 1951.

[5] K. Stewartson, "On the flow between two rotating coaxial discs," Mathematical Proceedings of the Cambridge Philosophical Society, vol. 49, no. 2, pp. 333-341, 1953.

[6] J. W. Daily and R. E. Nece, "Chamber dimension effects on induced flow and frictional resistance of enclosed rotating disks," Journal of Basic Engineering, vol. 82, pp. 217-232, 1960.

[7] M. Itoh, Y. Yamada, S. Imao, and M. Gonda, "Experimental on turbulent flow due an enclosed rotating disk," in Engineering Turbulence Modeling and Experiments, W. Rodi and E. N. Ganic, Eds., pp. 659-668, Elsevier, New York, NY, USA, 1990.

[8] A. J. Stepanoff, "Pompes centrifuges et pompes hélices," Transactions of the ASME, vol. 54, no. 15, pp. 334-352, 1932.

[9] A. Z. Szeri, A. Giron, S. J. Schneider, and H. N. Kaufman, "Flow between two rotating disks_-part 2: stability," Journal of Fluid Mechanics, vol. 134, pp. 133-154, 1983.

[10] D. Dijkstra and G. J. F. van Heijst, "The flow between two finite rotating disks enclosed by a cylinder," Journal of Fluid Mechanics, vol. 128, pp. 123-154, 1983.

[11] S. C. Cheah, H. Iacovides, D. C. Jackson, H. Ji, and B. E. Launder, "Experimental investigation of enclosed rotor-stator disk flows," Experimental Thermal and Fluid Science, vol. 9, no. 4, pp. 445-455, 1994.

[12] A. Randriamampianina, L. Elena, J. P. Fontaine, and R. Schiestel, "Numerical prediction of laminar, transitional and turbulent flows in shrouded rotor-stator systems," Physics of Fluids, vol. 9, no. 6, pp. 1696-1713, 1997.

[13] M. Djaoui, A. Malesys, and R. Debuchy, "Mise en évidence expérimentale de la sensibilité de l'écoulement de type rotorstator aux effets de bord," Comptes Rendus de l'Académie de Sciences. Serie IIb, vol. 327, no. 1, pp. 49-54, 1999.

[14] E. D’Haudt, S. Della Gatta, R. Debuchy, G. Bois, and F. Martelli, "Assessment of experimental and numerical flow investigation in rotating-disc systems," in Proceedings of the 11th International Symposium on Tranport Phenomena and Dynamics of Rotating Machinery (ISROMAC-11), Honolulu, Hawaii, USA, February-March 2006.

[15] R. Debuchy, S. Della Gatta, E. D’Haudt, G. Bois, and F. Martelli, "Influence of external geometrical modifications on the flow behaviour of a rotor-stator system: numerical and experimental investigation," Proceedings of the Institution of Mechanical Engineers, Part A: Journal of Power and Energy, vol. 221, no. 6, pp. 857-864, 2007. 
[16] R. Debuchy, A. Dyment, H. Muhe, and P. Micheau, "Radial inflow between a rotating and a stationary disc," European Journal of Mechanics, B/Fluids, vol. 17, no. 6, pp. 791-810, 1998.

[17] S. Poncet, Ecoulements de type rotor-stator soumis à un flux axial: de Batchelor à Stewartson, Ph.D. thesis, Université AixMarseille I, Marseille, France, 2005.

[18] S. Poncet, M. P. Chauve, and P. Le Gal, "Turbulent rotating disk flow with inward throughflow," Journal of Fluid Mechanics, vol. 522, pp. 253-262, 2005.

[19] R. Debuchy, Ecoulement turbulent avec aspiration radiale entre un disque fixe et un disque tournant, Ph.D. thesis, Université des Sciences et Technologies de Lille, Lille, France, 1993.

[20] S. Poncet, M.-P. Chauve, and R. Schiestel, "Batchelor versus Stewartson flow structures in a rotor-stator cavity with throughflow," Physics of Fluids, vol. 17, no. 7, Article ID 075110, 15 pages, 2005. 

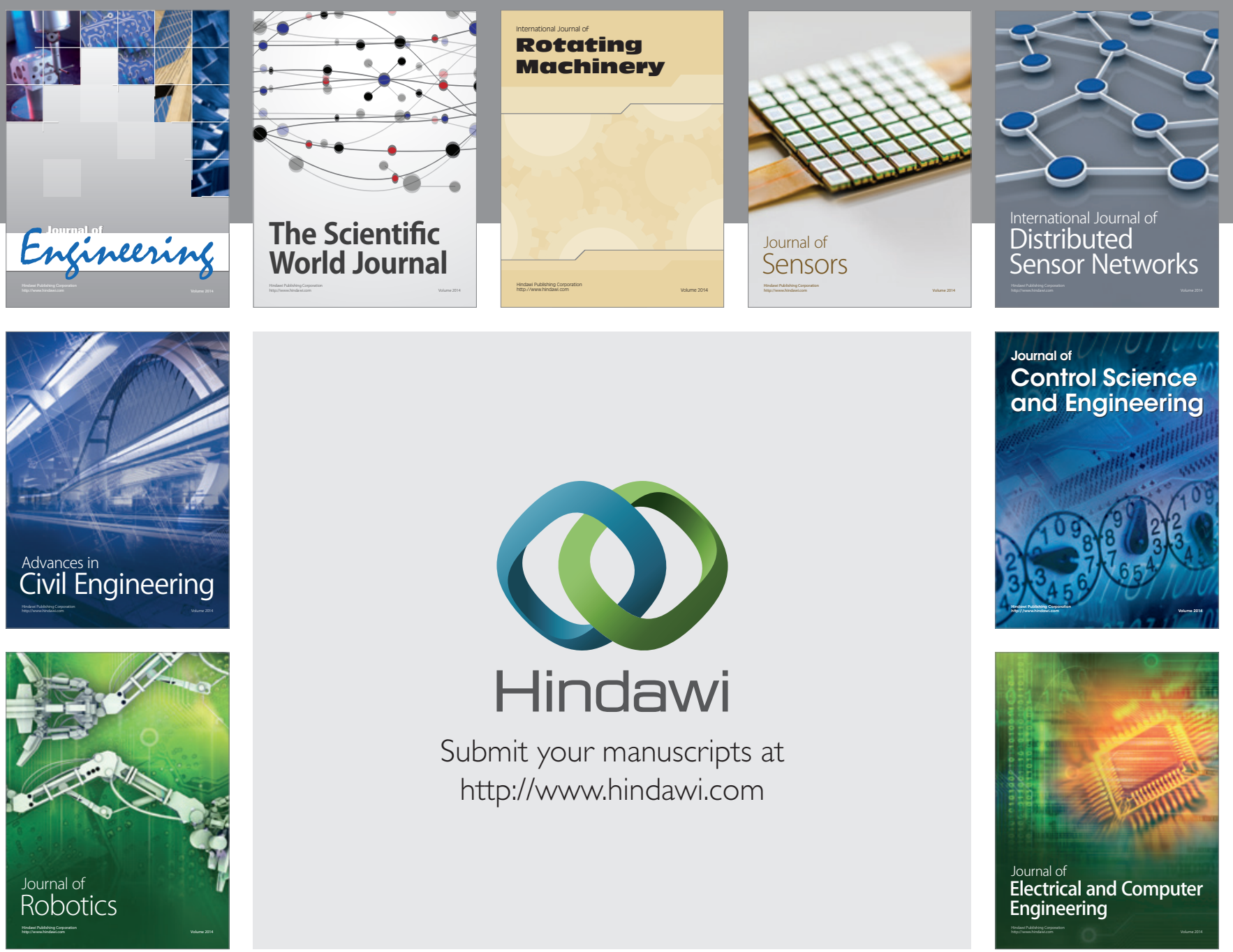

Submit your manuscripts at

http://www.hindawi.com
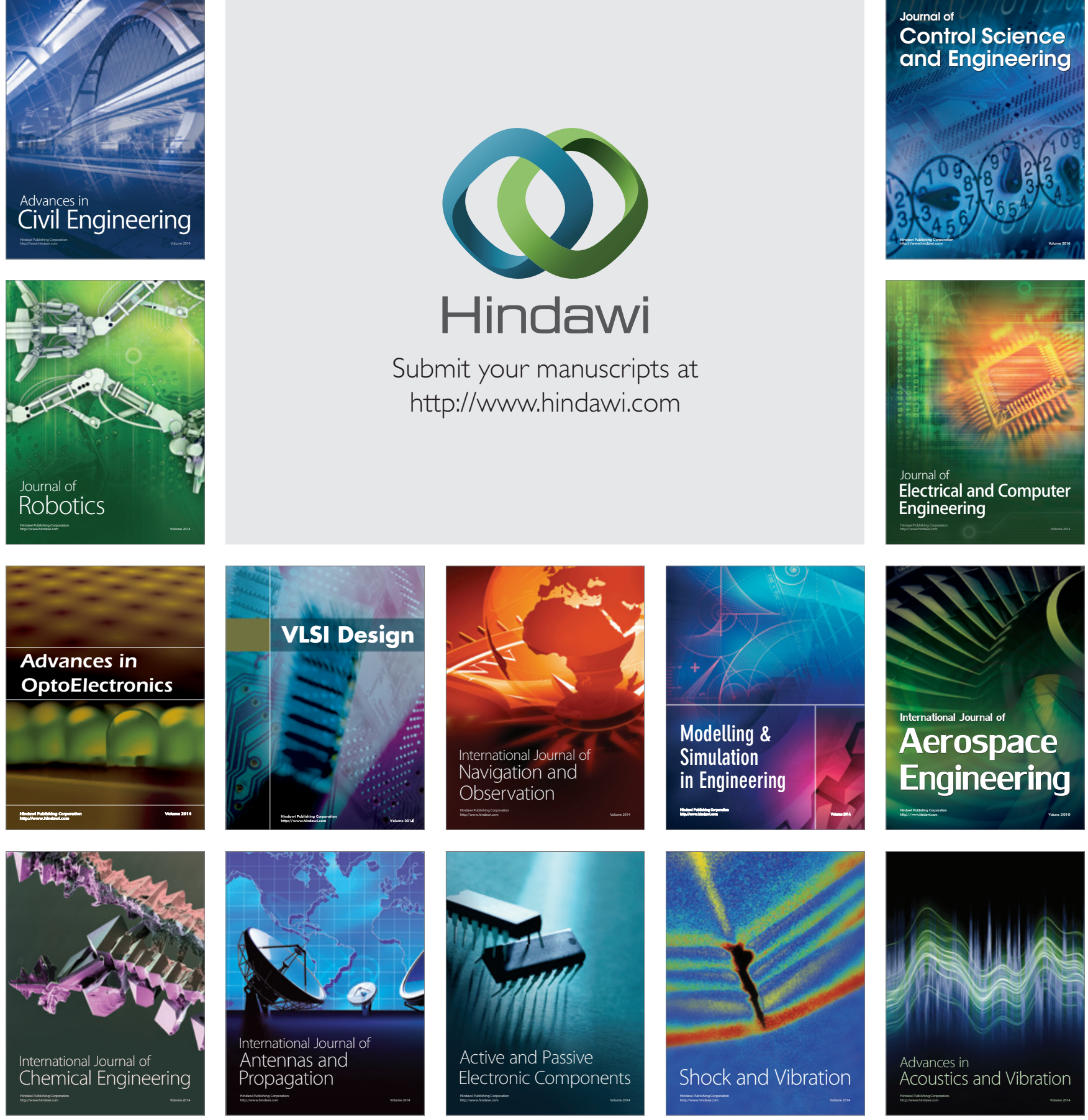